\title{
Exact Solutions for Nonlinear Transient Heat Transfer of Porous Fin Subjected to Magnetic Field with Variable Internal Heat Generation
}

\author{
M. G. Sobamowo \\ Department of Mechanical Engineering, Department of Mathematics, University of Lagos, Akoka, Lagos, Nigeria.
}

How to cite this paper: M. G. Sobamowo. (2020) Exact Solutions for Nonlinear Transient Heat Transfer of Porous Fin Subjected to Magnetic Field with Variable Internal Heat Generation. Journal of Applied Mathematics and Computation, 4(3), 94-103.

DOI: $10.26855 /$ jamc.2020.09.005

Received: June 22, 2020

Accepted: July 18, 2020

Published: August 19, 2020

"Corresponding author: M. G. Sobamowo, Department of Mechanical Engineering, Department of Mathematics, University of Lagos, Akoka, Lagos, Nigeria.

Email: mikegbeminiyiprof@yahoo.com

\begin{abstract}
This work establishes an exact analytical solution for the nonlinear transient thermal model of porous fin subjected to magnetic field with temperature-dependent internal heat generation. With the aid of Laplace transform method, the developed exact analytical model is used to study the impacts of the model parameters on the thermal performance of the fin. Through the developed symbolic heat transfer models using the exact analytical method, parametric studies show that increase in porosity, convective, radiative and magnetic parameters increase the rate of heat transfer from the fin and consequently improve the efficiency of the fin. It is established that the exact analytic solution can serve as basis for comparison of any other method of analysis of the problem and it can also provide platform for improvement in the design of porous fin in heat transfer equipment.
\end{abstract}

\section{Keywords}

Exact analytical solution, Magnetic field, Porous Fin, Nonlinear transient thermal model, Internal Heat Generation

\section{Introduction}

In the study of heat transfer, the past few decades as witnessed thermal analysis of extended surfaces which are used for effective cooling of thermal and electronics equipment. In such extended surfaces, porous fins have been used to enhance and augment the rate of heat transfer from the prime surfaces [1]. Consequently, numerous studies and extensive research on the use of the fins have been presented [2-18]. In the various research works, the developed heat transfer models are always nonlinear even for the simple model of natural convection dry or wet porous fin under the assumption of constant thermal and physical properties with no internal heat generation, radiation and magnetic field. The solutions of the nonlinear models have been presented using various approximate analytic and numerical methods such as Adomian decomposition method (ADM), spectral collocation method (SCM), homotopy perturbation method (HPM), homotopy analysis method (HAM), least square method (LSM), variational iterative method (VIM), differential transformation method (DTM) etc. [6-26]. The approximate analytic methods present explicit approximate analytical solutions which often involved complex mathematical analysis leading to analytic expressions involving large number terms. Furthermore, these methods are inherently with high computational cost and time accompanied with the requirement of high skills in mathematics. Moreover, in practice, analytical solutions with large number of terms and conditional statements for the solutions are not convenient for use by designers and engineers [27]. Consequently, in some other research works, recourse has been made to numerical methods in solving the nonlinear problems [2-4]. However, the classical way of finding exact analytical solution is obviously still very important since it serves as an 
accurate benchmark for numerical solutions. Exact analytical solutions for specified problems are also essential for the development of efficient applied numerical simulation tools. Inevitably, exact analytical expressions are required to show the direct relationship between the models parameters. When such exact analytical solutions are available, they provide good insights into the significance of various system parameters affecting the phenomena as it gives continuous physical insights than pure numerical or computation methods. It is convenient for parametric studies, accounting for the physics of the problem and appears more appealing than the numerical solutions. It helps in reducing the computation and simulation costs as well as the task involved in the analysis of real-life problems. Therefore, in this work, exact analytical solutions are developed for the nonlinear transient heat transfer equation of porous fin subjected to magnetic field with temperature-dependent internal heat generation using Laplace transforms. It is hope that the developed exact analytical model will serve as basis for comparison of any other method of analysis of the problem.

\section{Problem Formulation}

Consider a straight porous fin of temperature-dependent thermal conductivity $k(T)$, length $L$ and thickness $\delta$ that is exposed on both faces to a convective environment of moist air at temperature $T_{\infty}$ and with heat transfer co-efficient $h$ shown in Figure 1.

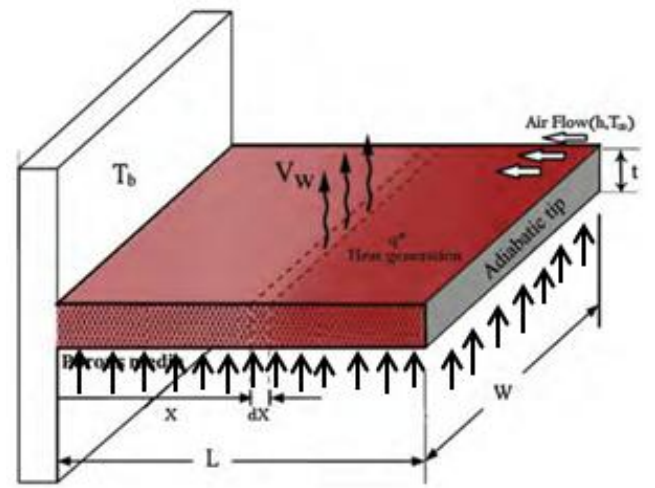

Magnetic field, $B_{y}$

Figure 1. Schematic of the longitudinal porous fin with the internal heat generation subjected to magnetic field.

A uniform magnetic field is applied in y-direction as depicted in figure. It is assumed that the porous medium is homogeneous, isotropic and saturated with a single-phase fluid and the physical properties of solid, as well as fluid are considered are constant. There exists thermal equilibrium between the fluid and the solid. One-dimensional heat transfer along the fin length is considered. Thermal contact resistance at the fin base is not considered and the fin tip is assumed adiabatic.

The thermal model is developed as

$$
\frac{\partial}{\partial x}\left(\left[1+\lambda\left(T-T_{\infty}\right)\right] \frac{d T}{d x}\right)-\frac{h\left(T-T_{\infty}\right)}{k_{e f f, 0} t}-\frac{\rho c_{p} g K \beta^{\prime}\left(T-T_{\infty}\right)^{2}}{k_{e f f, 0} t v_{f}}-\frac{\mathbf{J}_{c} \times \mathbf{J}_{c}}{\sigma k_{e f f, 0}}+\frac{q_{a}}{k_{e f f, 0}}\left[1+\psi\left(T-T_{\infty}\right)\right]=\frac{\rho c_{p}}{k_{e f f, 0}} \frac{\partial T}{\partial t}
$$

But

$$
\frac{\mathbf{J}_{c} \times \mathbf{J}_{c}}{\sigma k_{e f f, 0}}=\sigma B_{o}^{2} u^{2}
$$

Also, taking the magnetic flux to be linearly dependent on temperature, we have

$$
\frac{\partial}{\partial x}\left(\left[1+\lambda\left(T-T_{\infty}\right)\right] \frac{d T}{d x}\right)-\frac{h\left(T-T_{\infty}\right)}{k_{e f f, 0} t}-\frac{\rho c_{p} g K \beta^{\prime}\left(T-T_{\infty}\right)^{2}}{k_{e f f, 0} t v_{f}}-\frac{\sigma B_{o}^{2} u^{2}}{k_{e f f, 0} t}\left(T-T_{\infty}\right)+\frac{q_{a}}{k_{e f f}}\left[1+\psi\left(T-T_{\infty}\right)\right]=\frac{\rho c_{p}}{k_{e f f}} \frac{\partial T}{\partial t}
$$

On introducing the following dimensionless parameters in Eq. (3) into Eq. (4);

$$
\begin{aligned}
& X=\frac{x}{L}, \quad \theta=\frac{T-T_{\infty}}{T_{b}-T_{\infty}}, R a=G r \cdot \operatorname{Pr}=\left(\frac{\beta^{\prime} g T_{b} t^{3}}{v_{f}^{2}}\right)\left(\frac{\rho c_{p} v_{f}}{k_{e f f}}\right), \quad D a=\frac{K}{t^{2}}, \quad Q=\frac{q v_{f} t}{\rho \mathrm{c}_{p} \beta^{\prime} \mathrm{g} K\left(T_{b}-T_{\infty}\right)^{2}}, \\
& M^{2}=\frac{h L^{2}}{k_{\text {eff }} t}, S_{h}=\left(\frac{\beta^{\prime} g\left(T_{b}-T_{\infty}\right) t^{3}}{v_{f}^{2}}\right)\left(\frac{\rho c_{p} v_{f} K}{k_{e f f} t^{2}}\right) \frac{(L / t)^{2}}{k_{e f f}}=\frac{R a D a(L / t)^{2}}{k_{e f f}}, \quad \gamma=\psi\left(T_{b}-T_{\infty}\right), \quad H=\frac{\sigma B_{o}^{2} u^{2}}{t k_{e f f}}
\end{aligned}
$$


We arrived at the dimensionless governing differential Eq. (5) and the boundary conditions

$$
\frac{\partial^{2} \theta}{\partial X^{2}}+\beta \theta \frac{\partial^{2} \theta}{\partial X^{2}}+\beta\left(\frac{\partial \theta}{\partial X}\right)^{2}-M^{2} \theta-S_{h} \theta^{2}-H \theta+S_{h} Q \gamma \theta+S_{h} Q=\frac{\partial \theta}{\partial \tau}
$$

The initial and the boundary conditions in dimensionless forms are

$$
\begin{array}{ll}
\tau=0, & \theta=0, \quad 0<X<1 \\
\tau>0, & X=0, \quad \theta=1 \\
\tau>0, & X=1, \quad \frac{\partial \theta}{\partial x}=0
\end{array}
$$

\section{Development of exact analytical solutions}

In order to find exact analytical solution for the nonlinear transient equation as derived in Eq. (5), Laplace transform is apply to Eq. (5), which gives

$$
\frac{d^{2} \tilde{\theta}}{d X^{2}}+\beta \tilde{\theta} \frac{d^{2} \tilde{\theta}}{d X^{2}}+\beta\left(\frac{d \tilde{\theta}}{d X}\right)^{2}-s \tilde{\theta}-M^{2} \tilde{\theta}-S_{h} \tilde{\theta}^{2}-H \tilde{\theta}+S_{h} Q \gamma \tilde{\theta}+\frac{S_{h} Q}{s}=0
$$

And the boundary conditions in Laplace domain are

$$
\begin{aligned}
& s>0, \quad X=0, \quad \tilde{\theta}=\frac{1}{s} \\
& s>0, \quad X=1, \quad \frac{\partial \tilde{\theta}}{\partial x}=0
\end{aligned}
$$

taking the transformation

$$
\frac{d \tilde{\theta}}{d X}=\tilde{\phi}
$$

which implies

$$
\frac{d^{2} \tilde{\theta}}{d X^{2}}=\frac{d \tilde{\phi}}{d X}=\tilde{\phi} \frac{d \tilde{\phi}}{d \tilde{\theta}}
$$

If we substitute Eq. (20) and (21) into Eq. (16), we have

$$
\tilde{\phi} \frac{d \tilde{\phi}}{d \tilde{\theta}}+\beta \tilde{\theta} \tilde{\phi} \frac{d \tilde{\phi}}{d \tilde{\theta}}+\beta \tilde{\phi}^{2}-s \tilde{\theta}-M^{2} \tilde{\theta}-S_{h} \tilde{\theta}^{2}-H \tilde{\theta}+S_{h} Q \gamma \tilde{\theta}+\frac{S_{h} Q}{s}=0
$$

The above equation can easily be written as

$$
(1+\beta \tilde{\theta}) \tilde{\phi} d \tilde{\phi}+\left\{\beta \tilde{\phi}^{2}-s \tilde{\theta}-M^{2} \tilde{\theta}-S_{h} \tilde{\theta}^{2}-H \tilde{\theta}+S_{h} Q \gamma \tilde{\theta}+\frac{S_{h} Q}{s}\right\} d \tilde{\theta}=0
$$

If we multiply Eq. (12) through by

$$
(1+\beta \tilde{\theta})^{2} \tilde{\phi} d \tilde{\phi}+(1+\beta \tilde{\theta})\left\{\beta \tilde{\phi}^{2}-s \tilde{\theta}-M^{2} \tilde{\theta}-S_{h} \tilde{\theta}^{2}-H \tilde{\theta}+S_{h} Q \gamma \tilde{\theta}+\frac{S_{h} Q}{s}\right\} d \tilde{\theta}=0
$$

The above Eq. (13) is a total differential equation which has a solution of the form

$$
\frac{1}{2}(1+\beta \tilde{\theta})^{2} \tilde{\phi}^{2}-\beta S_{h} \frac{\theta^{4}}{4}+S_{h}\left(\beta Q \gamma-\beta s-M^{2}-H^{2}-1\right) \frac{\theta^{3}}{3}+\left\{S_{h} Q\left(\frac{\beta}{s}+\gamma\right)-\left(s+M^{2}+H\right)\right\} \frac{\theta^{2}}{2}+\frac{S_{h} Q}{s} \theta=C
$$

where $\mathrm{C}$ is the constant of integration

$$
\text { Recall that } \tilde{\phi}=\frac{d \tilde{\theta}}{d X} \quad \rightarrow \tilde{\phi}^{2}=\left(\frac{d \tilde{\theta}}{d X}\right)^{2}
$$

Therefore, Eq. (14) can be written as

$$
\frac{1}{2}(1+\beta \tilde{\theta})^{2}\left(\frac{d \tilde{\theta}}{d X}\right)^{2}-\beta S_{h} \frac{\tilde{\theta}^{4}}{4}+S_{h}\left(\beta Q \gamma-\beta s-M^{2}-H^{2}-1\right) \frac{\tilde{\theta}^{3}}{3}+\left\{S_{h} Q\left(\frac{\beta}{s}+\gamma\right)-\left(s+M^{2}+H\right)\right\} \frac{\tilde{\theta}^{2}}{2}+\frac{S_{h} Q}{s} \tilde{\theta}=C
$$

with the application of the first boundary condition, 


$$
X=1, \quad \frac{d \tilde{\theta}}{d X}=0 \quad \rightarrow X=1, \quad \tilde{\theta}=\tilde{\theta}_{o},
$$

We found constant $\mathrm{C}$ as

$$
-\beta S_{h} \frac{\tilde{\theta}_{0}^{4}}{4}+S_{h}\left(\beta Q \gamma-\beta s-M^{2}-H^{2}-1\right) \frac{\tilde{\theta}_{0}^{3}}{3}+\left\{S_{h} Q\left(\frac{\beta}{s}+\gamma\right)-\left(s+M^{2}+H\right)\right\} \frac{\tilde{\theta}_{0}^{2}}{2}+\frac{S_{h} Q}{s} \tilde{\theta}_{0}=C
$$

where $\tilde{\theta}_{o}$ is the dimensionless temperature (in Laplace domain) at the tip

On substituting Eq. (16) into Eq. (15), we have

After arrangement

$$
\begin{aligned}
& \frac{1}{2}(1+\beta \tilde{\theta})^{2}\left(\frac{d \tilde{\theta}}{d X}\right)^{2}+\beta S_{h}\left(\frac{\tilde{\theta}_{0}^{4}}{4}-\frac{\tilde{\theta}^{4}}{4}\right)-S_{h}\left(\beta Q \gamma-\beta s-M^{2}-H^{2}-1\right)\left(\frac{\tilde{\theta}_{0}^{3}}{3}-\frac{\tilde{\theta}^{3}}{3}\right) \\
& +\left\{S_{h} Q\left(\frac{\beta}{s}+\gamma\right)-\left(s+M^{2}+H\right)\right\}\left(\frac{\tilde{\theta}_{0}^{2}}{4}-\frac{\tilde{\theta}^{2}}{4}\right)-\frac{S_{h} Q}{s}\left(\tilde{\theta}_{0}-\theta\right)=0
\end{aligned}
$$

$$
\begin{aligned}
& \frac{1}{2}(1+\beta \tilde{\theta})^{2}\left(\frac{d \tilde{\theta}}{d X}\right)^{2}=S_{h}\left(\beta Q \gamma-\beta s-M^{2}-H^{2}-1\right)\left(\frac{\tilde{\theta}_{0}^{3}}{3}-\frac{\tilde{\theta}^{3}}{3}\right)+\frac{S_{h} Q}{s}\left(\tilde{\theta}_{0}-\theta\right) \\
& +\left\{S_{h} Q\left(\frac{\beta}{s}+\gamma\right)-\left(s+M^{2}+H\right)\right\}\left(\frac{\tilde{\theta}_{0}^{2}}{4}-\frac{\tilde{\theta}^{2}}{4}\right)-\beta S_{h}\left(\frac{\tilde{\theta}_{0}^{4}}{4}-\frac{\tilde{\theta}^{4}}{4}\right)
\end{aligned}
$$

A further rearrangement gives

$$
\sqrt{2} d X=\frac{\left|(1+\beta \tilde{\theta})^{2}\right| d \tilde{\theta}}{\sqrt{\left\{\begin{array}{l}
S_{h}\left(\beta Q \gamma-\beta s-M^{2}-H^{2}-1\right)\left(\frac{\tilde{\theta}_{0}^{3}}{3}-\frac{\tilde{\theta}^{3}}{3}\right)+\frac{S_{h} Q}{s}\left(\tilde{\theta}_{0}-\theta\right) \\
+\left\{S_{h} Q\left(\frac{\beta}{s}+\gamma\right)-\left(s+M^{2}+H\right)\right\}\left(\frac{\tilde{\theta}_{0}^{2}}{4}-\frac{\tilde{\theta}^{2}}{4}\right)-\beta S_{h}\left(\frac{\tilde{\theta}_{0}^{4}}{4}-\frac{\tilde{\theta}^{4}}{4}\right)
\end{array}\right\}}}
$$

Integration of both sides of the Eq. (19) gives

$$
\begin{aligned}
& \sqrt{2} \int_{0}^{X} d X=\int_{\theta_{0}}^{\theta} \frac{\left|(1+\beta \tilde{\theta})^{2}\right| d \tilde{\theta}}{\sqrt{\left\{\begin{array}{l}
S_{h}\left(\beta Q \gamma-\beta s-M^{2}-H^{2}-1\right)\left(\frac{\tilde{\theta}_{0}^{3}}{3}-\frac{\tilde{\theta}^{3}}{3}\right)+\frac{S_{h} Q}{s}\left(\tilde{\theta}_{0}-\theta\right) \\
+\left\{S_{h} Q\left(\frac{\beta}{s}+\gamma\right)-\left(s+M^{2}+H\right)\right\}\left(\frac{\tilde{\theta}_{0}^{2}}{4}-\frac{\tilde{\theta}^{2}}{4}\right)-\beta S_{h}\left(\frac{\tilde{\theta}_{0}^{4}}{4}-\frac{\tilde{\theta}^{4}}{4}\right)
\end{array}\right\}}} \\
& \sqrt{2} X=\int_{\theta_{0}}^{\theta} \frac{\left|(1+\beta \tilde{\theta})^{2}\right| d \tilde{\theta}}{\sqrt{\left\{\begin{array}{l}
S_{h}\left(\beta Q \gamma-\beta s-M^{2}-H^{2}-1\right)\left(\frac{\tilde{\theta}_{0}^{3}}{3}-\frac{\tilde{\theta}^{3}}{3}\right)+\frac{S_{h} Q}{s}\left(\tilde{\theta}_{0}-\theta\right) \\
+\left\{S_{h} Q\left(\frac{\beta}{s}+\gamma\right)-\left(s+M^{2}+H\right)\right\}\left(\frac{\tilde{\theta}_{0}^{2}}{4}-\frac{\tilde{\theta}^{2}}{4}\right)-\beta S_{h}\left(\frac{\tilde{\theta}_{0}^{4}}{4}-\frac{\tilde{\theta}^{4}}{4}\right)
\end{array}\right\}}}
\end{aligned}
$$

suppose that

$$
G\left(\tilde{\theta} ; S_{h}, M, H, Q, \tilde{\theta}_{o}\right)=G\left(\theta ; S_{h}, N, Q, \theta_{o}\right)=\int_{\theta_{0}}^{\theta} \frac{\left|(1+\beta \tilde{\theta})^{2}\right| d \tilde{\theta}}{\left.\sqrt{\left\{\begin{array}{l}
S_{h}\left(\beta Q \gamma-\beta s-M^{2}-H^{2}-1\right)\left(\frac{\tilde{\theta}_{0}^{3}}{3}-\frac{\tilde{\theta}^{3}}{3}\right)+\frac{S_{h} Q}{s}\left(\tilde{\theta}_{0}-\theta\right) \\
+\left\{S_{h} Q\left(\frac{\beta}{s}+\gamma\right)-\left(s+M^{2}+H\right)\right\}
\end{array}\right\}\left(\frac{\tilde{\theta}_{0}^{2}}{4}-\frac{\tilde{\theta}^{2}}{4}\right) \mid-\beta S_{h}\left(\frac{\tilde{\theta}_{0}^{4}}{4}-\frac{\tilde{\theta}^{4}}{4}\right)}\right\}}
$$


where $N=M^{2}+H$

The integral in Eq. (20) is expressible (e.g. via Wolfram's Mathematica) in term of incomplete elliptic integrals of the first kind. For instant [28]

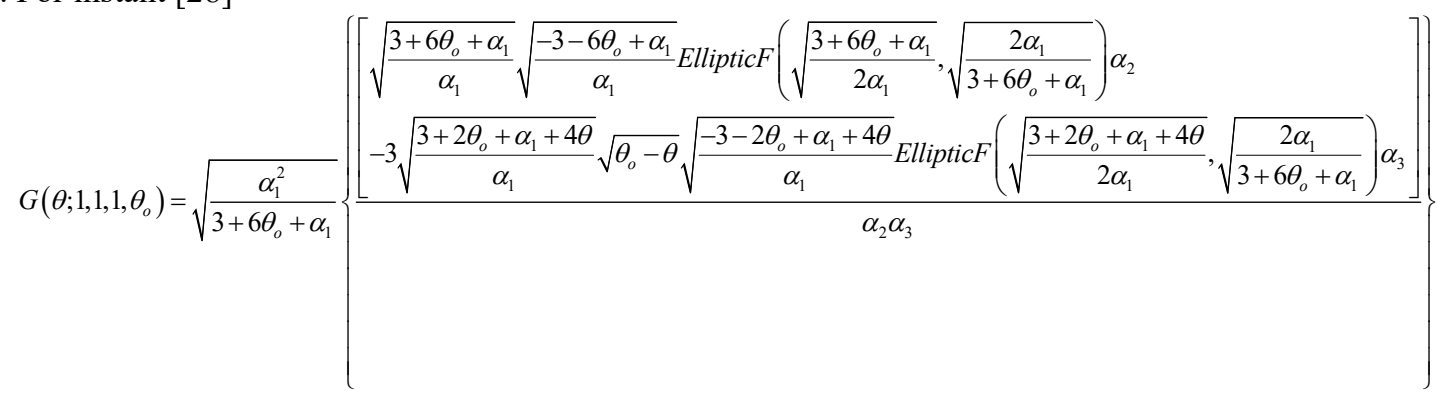

where

$$
\begin{aligned}
& \alpha_{1}=\sqrt{57-12 \theta_{o}-12 \theta_{o}^{2}} \\
& \alpha_{2}=\sqrt{6 \theta^{3}-18 \theta+9 \theta^{2}-6 \theta_{o}^{3}+18 \theta_{o}-9 \theta_{o}^{2}} \\
& \alpha_{3}=\sqrt{2-2 \theta_{o}-2 \theta_{o}^{2}}
\end{aligned}
$$

Therefore, the exact solution of Eq. (5) in implicit form is given by

$$
X=G\left(\tilde{\theta} ; S_{h}, N, Q, \tilde{\theta}_{o}\right)
$$

Where the unknown $\tilde{\theta}_{o}$ in the solution can be determined from the second boundary condition

$$
X=0, \quad \theta=1 \rightarrow 0=G\left(1 ; S_{h}, N, Q, \tilde{\theta}_{o}\right) \rightarrow G\left(1 ; S_{h}, N, Q, \tilde{\theta}_{o}\right)=0
$$

i.e. for any given $S_{h}, N$, and $Q, \tilde{\theta}_{o}$ is obtained from

$$
G\left(1 ; S_{h}, N, Q, \tilde{\theta}_{o}\right)=0
$$

And Elliptic F in Eq. (22) is the incomplete elliptic integral of the first kind defined as

$$
\operatorname{EllipticF}(X, K)=\int_{0}^{X} \frac{d \tau}{\sqrt{1-\tau^{2}} \sqrt{1-K^{2} \tau^{2}}}
$$

The elliptic function can be exactly and analytically evaluated as follows

$$
\text { Let } \quad \tau=\sin \vartheta, \quad x=\sin \phi
$$

Then Eq. (26) becomes

$$
\operatorname{EllipticF}(\phi, K)=\int_{0}^{\phi} \frac{d \vartheta}{\sqrt{1-K^{2} \sin \vartheta}}
$$

In order to evaluate the integral, we expand the integral in the form

$$
\frac{1}{\sqrt{1-K^{2} \sin ^{2} \vartheta}}=1+\frac{K^{2}}{2} \sin ^{2} \vartheta+\frac{3 K^{4}}{8} \sin ^{4} \vartheta+\frac{5 K^{6}}{16} \sin ^{6} \vartheta+\frac{35 K^{8}}{128} \sin ^{8} \vartheta+\ldots
$$

Eq. (40) be written as

$$
\frac{1}{\sqrt{1-K^{2} \sin ^{2} \vartheta}}-1=\frac{K^{2}}{2} \sin ^{2} \vartheta+\frac{3 K^{4}}{8} \sin ^{4} \vartheta+\frac{5 K^{6}}{16} \sin ^{6} \vartheta+\frac{35 K^{8}}{128} \sin ^{8} \vartheta+\ldots+\left(\prod_{n=1}^{\infty} \frac{2 n-1}{2 n}\right) K^{2 n} \sin ^{2 n} \vartheta
$$

Generally, we can write

$$
\frac{1}{\sqrt{1-K^{2} \sin ^{2} \vartheta}}=1+\sum_{n=1}^{\infty}\left(\prod_{n=1}^{\infty} \frac{2 n-1}{2 n}\right) K^{2 n} \sin ^{2 n} \vartheta
$$


The above series is uniformly convergent for all $\vartheta$, and may, therefore, be integrated term by term. Then, we have

$$
\text { EllipticF }(\phi, K)=\int_{0}^{\phi}\left\{1+\sum_{n=1}^{\infty}\left(\prod_{n=1}^{\infty} \frac{2 n-1}{2 n}\right) K^{2 n} \sin ^{2 n} \vartheta\right\} d \vartheta
$$

But

Therefore

$$
\begin{aligned}
& \int \sin ^{2 n} \vartheta d \vartheta=\frac{-\cos \vartheta}{2 n}\left\{\sin ^{2 n-1} \vartheta+\sum_{k=1}^{n-1} \frac{(2 n-1)(2 n-3) \ldots(2 n-2 k+1)}{2^{k}(n-1)(n-2) \ldots(n-k)} \sin ^{2 n-2 k-1} \vartheta\right\} \\
& +\frac{(2 n-1) ! !}{2^{n} n !} \vartheta
\end{aligned}
$$

$$
\text { EllipticF }(\phi, K)=\left\{\phi+\sum_{n=1}^{\infty}\left(\prod_{n=1}^{\infty} \frac{2 n-1}{2 n}\right) K^{2 n}\left\{\begin{array}{l}
\frac{-\cos \phi}{2 n}\left\{\sin ^{2 n-1} \phi+\sum_{k=1}^{n-1} \frac{(2 n-1)(2 n-3) \ldots(2 n-2 k+1)}{2^{k}(n-1)(n-2) \ldots(n-k)} \sin ^{2 n-2 k-1} \phi\right. \\
+\frac{(2 n-1) ! !}{2^{n} n !} \phi
\end{array}\right\}\right\}
$$

The symbolic and numerical calculations involved in the function $G\left(\theta ; S_{h}, N, Q, \tilde{\theta}_{o}\right)$ are carried out via Wolfram's Mathematica.

The determination of the inverse Laplace transforms of Eq. (23) is very complex. However, they can be numerically evaluated using Simon's approach [39] given as

$$
\theta(X, \tau)=\frac{e^{a_{p} \tau}}{\tau}\left[\frac{1}{2} \tilde{\theta}\left(X, a_{p}\right)+\sum_{n=1}^{N} \operatorname{Re}\left[\tilde{\theta}\left(X, a_{p}+i \frac{n \pi}{\tau}\right)\right](-1)^{n}\right]
$$

Lee et al. [39] suggested values of $a_{p} \tau$ ranging between 4 and 5. Eq. (34) converges more quickly because it does not contain oscillating cosine and sine functions

Where the optimally value is [40]

$$
a_{p} \tau=4.7 \Rightarrow a_{p}=\frac{4.7}{\tau}
$$

Alternatively, Durbin's method can be used for find the inverse Laplace transform as follows

$$
\theta(X, \tau)=\frac{2 e^{\frac{a_{v} \tau}{N}}}{\tau}\left[\frac{-1}{2} \operatorname{Re}\left\{\tilde{\theta}\left(X, a_{v}\right)\right\}+\operatorname{Re} \sum_{n=1}^{N-1}[\Upsilon(X, n)+i \Phi(X, n)] e^{\frac{i 2 \pi}{N}}\right]
$$

where

$$
\begin{aligned}
& \Upsilon(X, n)=\sum_{m=1}^{M} \operatorname{Re}\left\{\tilde{\theta}\left(X, a_{v}+i(k+m N) \frac{2 \pi}{\tau}\right)\right\} \\
& \Phi(X, n)=\sum_{m=1}^{M} \operatorname{Im}\left\{\tilde{\theta}\left(X, a_{v}+i(k+m N) \frac{2 \pi}{\tau}\right)\right\}
\end{aligned}
$$

Choosing $a$ constant value in inverse Laplace transform is explained by Dublin. According to Durbin's study, the value of $a_{v} \tau$ was set value between 5 and 10 to getting better result. Terminally, the solutions are multiplied with Lanczos $L k$ factor and best solutions are obtained.

$$
L_{k}=\frac{\sin \left(\frac{k \pi}{N}\right)}{\left(\frac{k \pi}{N}\right)}
$$




\section{Results and Discussion}

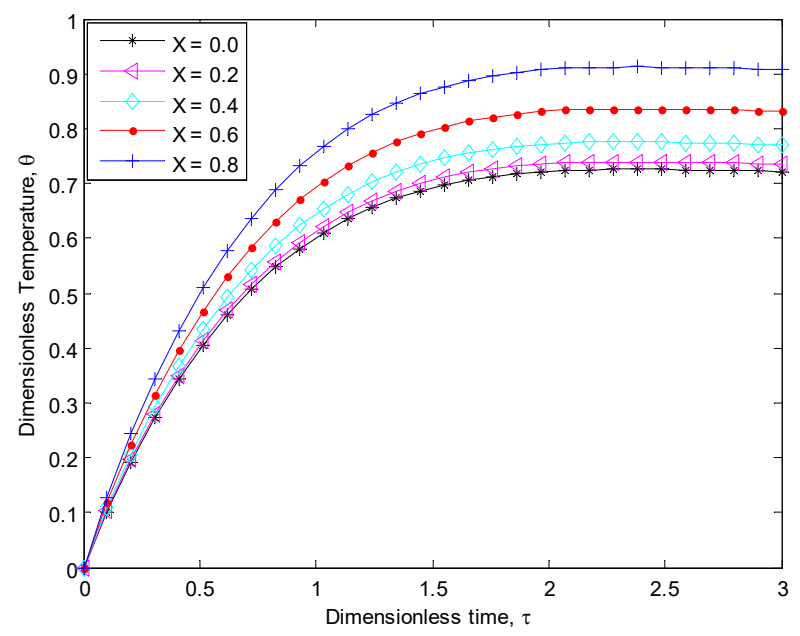

Figure 2. Dimensionless Temperature history at different points in the fin when $M=0.4, H=0.09, S=0.5 ; Q=0.4, G=0.2$.

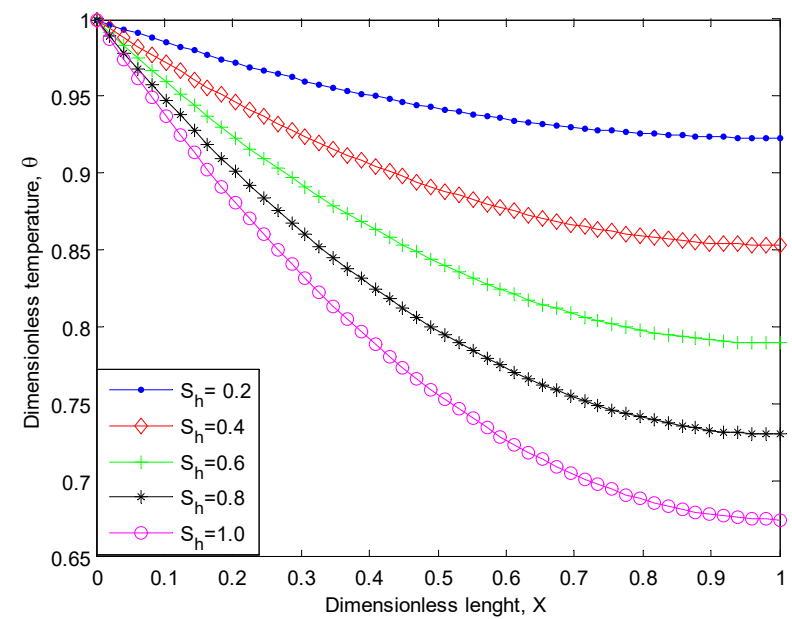

Figure 3. Effects of porous parameter on the dimensionless temperature distribution in the fin when $M=0.4, H=0.09, S=0.5$; $\mathrm{Q}=\mathbf{0 . 4}, \mathbf{G}=\mathbf{0 . 2}$.

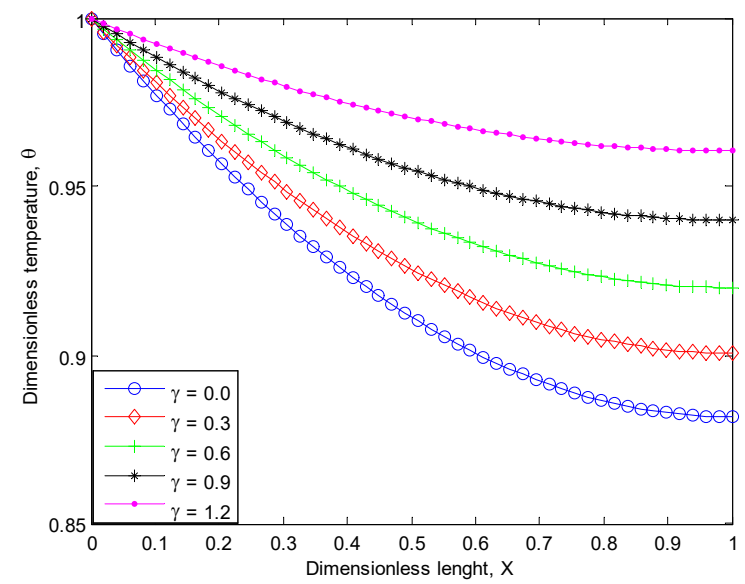

Figure 4. Effects of temperature-dependent internal heat generation parameter on the dimensionless temperature distribution in the fin when $\mathrm{M}=0.4, \mathrm{H}=0.09, \mathrm{~S}=0.5 ; \mathrm{Q}=0.4, \mathrm{G}=0.2$. 


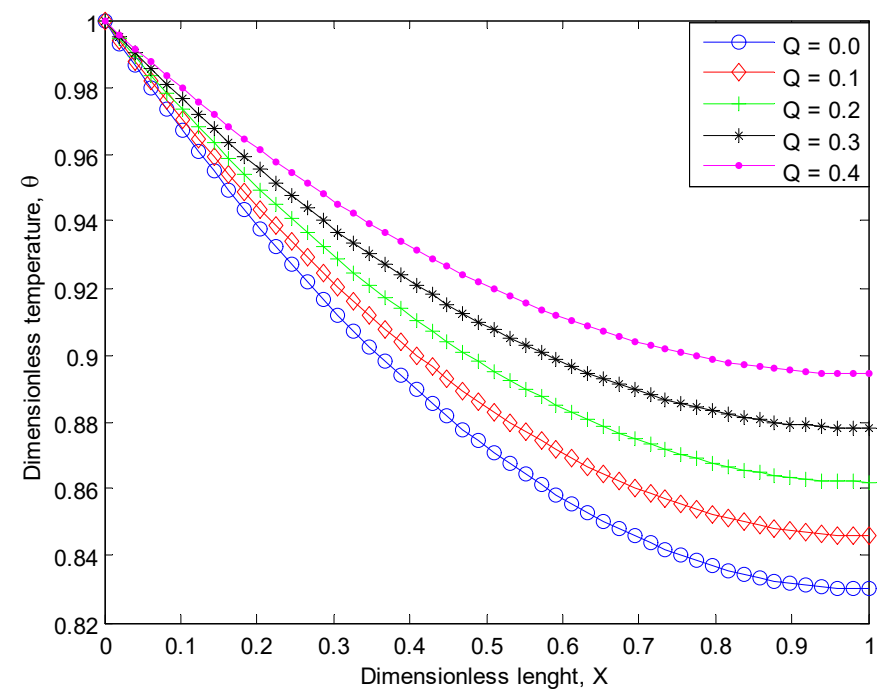

Figure 5. Effects of internal heat generation parameter on the dimensionless temperature distribution in the fin when $M=0.4$, $\mathrm{H}=0.09, \mathrm{~S}=0.5 ; \mathrm{Q}=0.4, \mathrm{G}=0.2$.

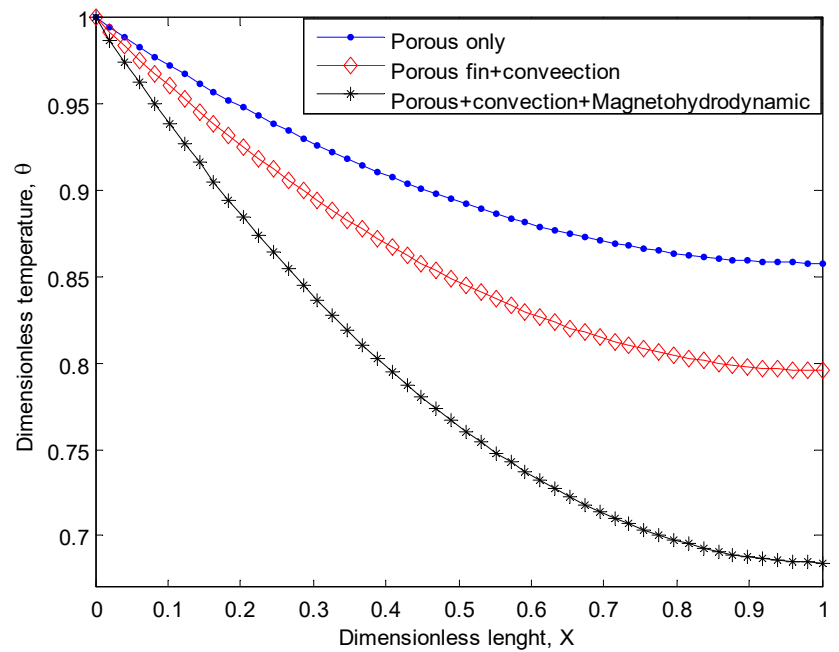

Figure 6. Effects of porosity, convection and magnetohydrodynamic on the dimensionless temperature distribution in the fin when $\mathrm{M}=1.0, \mathrm{H}=4.0, \mathrm{~S}=0.5 ; \mathrm{Q}=0.4, \mathrm{G}=0.2$.

Figure 2 shows dimensionless temperature histories at different locations along the fin length while Figures 3-6 show the effects of parameters on the dimensionless temperature distribution of the porous fin and by extension on the rate of heat transfer. From the figures, it is shown that as the convective, internal heat generation, magnetic and non-linear porous parameter has significant effects on the heat transfer in the porous fin. The results depicted that as the non-linear term porous parameter increases, the dimensionless temperature distribution in the fin decreases and consequently, the rate of heat transfer by the fin increases (Figure 2) while rate of heat transfer decreases with increase in internal heat generation (Figures 3-4). Also, the dimensionless temperature distribution in the fin decreases which implies that the rate of heat by the fin increases as the convective and magnetic parameter increase (Figures 5-6).

\section{Conclusion}

In this work, exact analytical solution is developed for the nonlinear transient heat transfer equation of porous fin subjected to magnetic field with temperature-dependent internal heat generation using Laplace transform method. Parametric studies were carried out using the developed exact analytical solutions. In the study, it was established that increase in porosity, convective, radiative and magnetic parameters increase the rate of heat transfer from the fin and consequently improve the efficiency of the fin. The developed exact analytical model can serve as basis for comparison 
of any other method of analysis of the problem and they also provide platform for the exact analysis and improvement in the design of porous fin in heat transfer equipment.

\section{Nomenclature}

$\mathrm{A}_{\mathrm{c}} \quad$ cross sectional area of the fins

$\mathrm{A}_{\mathrm{p}} \quad$ profile area of the fins

B Magnetic induction

Bi Biot number

$\mathrm{c}_{\mathrm{pa}}$ specific heat capacity

E Electric field

$\mathrm{h}$ convective heat transfer coefficient

$\mathrm{H}$ Hartman number

$\mathrm{J}_{\mathrm{c}} \quad$ conduction current intensity

Jtotal current intensity

$\mathrm{k} \quad$ thermal conductivity of the fin material

$\mathrm{k}_{\mathrm{a}}$ thermal conductivity of the fin material at ambient temperature

$\mathrm{k}_{\mathrm{b}}$ thermal conductivity of the fin material at the base temperature of the fin

$\mathrm{L} \quad$ Length of the fin

M dimensionless thermo-geometric fin parameter

$\mathrm{m}$ thermo-geometric fin parameter

$P \quad$ perimeter of the fin

q rate of heat transfer

Q dimensionless heat transfer

$t$ thickness of the fin

$\mathrm{T}$ Temperature

$\mathrm{T}_{\mathrm{a}} \quad$ ambient temperature

$\mathrm{T}_{b} \quad$ Temperature at the base of the fin

$\mathrm{x}$ fin axial distance, $\mathrm{m}$

$\mathrm{X}$ dimensionless length of the fin

$u, V$ velocity

\section{Greek Symbols}

$\sigma \quad$ conductivity of the medium

$\theta \quad$ dimensionless temperature

$\theta_{\mathrm{b}} \quad$ dimensionless temperature at the base of the fin

$\rho$ density of the fin

\section{References}

[1] S. Kiwan, A. Al-Nimr. (2001). Using Porous Fins for Heat Transfer Enhancement. ASME J. Heat Transfer, 2001, $123: 790-5$.

[2] S. Kiwan. (2007a). Effect of radiative losses on the heat transfer from porous fins. Int. J. Therm. Sci., 46, $1046-1055$.

[3] S. Kiwan. (2007b). Thermal analysis of natural convection porous fins. Tran. Porous Media, 67, 17-29.

[4] S. Kiwan, O. Zeitoun. (2008). Natural convection in a horizontal cylindrical annulus using porous fins. Int. J. Numer. Heat Fluid Flow, 18(5), 618-634.

[5] R. S. Gorla, A. Y. Bakier. (2011). Thermal analysis of natural convection and radiation in porous fins. Int. Commun. Heat Mass Transfer, 38, 638-645.

[6] B. Kundu, D. Bhanji. (2011). An analytical prediction for performance and optimum design analysis of porous fins. Int. J. Refrigeration, 34, 337-352.

[7] B. Kundu, D. Bhanja, K. S. Lee. (2012). A model on the basis of analytics for computing maximum heat transfer in porous fins. Int. J. Heat Mass Transfer, 55(25-26), 7611-7622.

[8] A. Taklifi, C. Aghanajafi, H. Akrami. (2010). The effect of MHD on a porous fin attached to a vertical isothermal surface. Transp Porous Med., 85, 215-31.

[9] D. Bhanja, B. Kundu. (2011). Thermal analysis of a constructal T-shaped porous fin with radiation effects. Int J Refrigerat, 34, $1483-96$.

[10] B. Kundu. (2007). Performance and optimization analysis of SRC profile fins subject to simultaneous heat and mass transfer. Int. J. Heat Mass Transfer, 50, 1545-1558. 
[11] S. Saedodin, S. Sadeghi. (2013). Temperature distribution in long porous fins in natural convection condition. Middle-east J. Sci. Res., 13(6), 812-817.

[12] S. Saedodin, M. Olank. (2011). Temperature Distribution in Porous Fins in Natural Convection Condition. Journal of American Science, 7(6), 476-481.

[13] M. T. Darvishi, R. Gorla, R. S., Khani, F., Aziz, A.-E. (2015). Thermal performance of a porus radial fin with natural convection and radiative heat losses. Thermal Science, 19(2), 669-678.

[14] M. Hatami, D. D. Ganji. (2013). Thermal performance of circular convective-radiative porous fins with different section shapes and materials. Energy Conversion and Management, 76, 185-193.

[15] M. Hatami, D. D. Ganji. (2014). Thermal behavior of longitudinal convective-radiative porous fins with different section shapes and ceramic materials ( $\mathrm{SiC}$ and $\mathrm{Si} 3 \mathrm{~N} 4$ ). International of J. Ceramics International, 40, 6765-6775.

[16] M. Hatami, A. Hasanpour, D. D. Ganji. (2013). Heat transfer study through porous fins (Si3N4 and AL) with temperature-dependent heat generation. Energ. Convers. Manage, 74, 9-16.

[17] M. Hatami, D. D. Ganji. (2014). Investigation of refrigeration efficiency for fully wet circular porous fins with variable sections by combined heat and mass transfer analysis. International Journal of Refrigeration, 40, 140-151.

[18] M. Hatami, G. H. R. M. Ahangar, D. D. Ganji, K. Boubaker. (2014). Refrigeration efficiency analysis for fully wet semi-spherical porous fins. Energy Conversion and Management, 84, 533-540.

[19] R. Gorla, R. S., Darvishi, M. T. Khani, F. (2013). Effects of variable Thermal conductivity on natural convection and radiation in porous fins. Int. Commun. Heat Mass Transfer, 38, 638-645.

[20] A. Moradi, T. Hayat and A. Alsaedi. (2014). Convective-radiative thermal analysis of triangular fins with temperature-dependent thermal conductivity by DTM. Energy Conversion and Management, 77, 70-77.

[21] S. Saedodin. M. Shahbabaei. (2013). Thermal Analysis of Natural Convection in Porous Fins with Homotopy Perturbation Method (HPM). Arab J Sci Eng., 38: 2227-2231.

[22] H. Ha, Ganji D. D., and Abbasi M. (2005). Determination of Temperature Distribution for Porous Fin with Temperature-Dependent Heat Generation by Homotopy Analysis Method. J Appl Mech Eng., 4(1).

[23] H. A. Hoshyar, I. Rahimipetroudi, D. D. Ganji, A. R. Majidian. (2015). Thermal performance of porous fins with temperature-dependent heat generation via Homotopy perturbation method and collocation method. Journal of Applied Mathematics and Computational Mechanics, 14(4), 53-65.

[24] Y. Rostamiyan, D. D. Ganji, I. R. Petroudi, and M. K. Nejad. (2014). Analytical Investigation of Nonlinear Model Arising in Heat Transfer Through the Porous Fin. Thermal Science, 18(2), 409-417.

[25] S. E. Ghasemi, P. Valipour, M. Hatami, D. D. Ganji. (2014). Heat transfer study on solid and porous convective fins with temperature-dependent heat-generation using efficient analytical method. J. Cent. South Univ., 21, 4592-4598.

[26] I. R. Petroudi, D. D. Ganji, A. B. Shotorban, M. K. Nejad, E. Rahimi, R. Rohollahtabar and F. Taherinia. (2012). Semi-Analytical Method for Solving Nonlinear Equation Arising in Natural Convection Porous fin. Thermal Science, 16(5), 1303-1308.

[27] M. G. Sobamowo. (2016). Thermal analysis of longitudinal fin with temperature-dependent properties and internal heat generation using Galerkin's method of weighted residual. Applied Thermal Engineering, 99, 1316-1330.

[28] S. Abbasbandy, E. Shivanian, and I. Hashim. (2011). Exact analytical solution of a forced convection in porous-saturated duct. Comm. Nonlinear Sci Numer Simulat. 16, 3981-3989. 